\title{
Flipping the Electromagnetic Theory classroom
}

\section{Andrew Berger}

Andrew J. Berger, "Flipping the Electromagnetic Theory classroom," Proc. SPIE 10452, 14th Conference on Education and Training in Optics and Photonics: ETOP 2017, 104522N (16 August 2017); doi: 10.1117/12.2269783

SPIE Event: 14th Conference on Education and Training in Optics and Photonics, SPIE. ETOP 2017, 2017, Hangzhou, China 


\title{
Flipping the Electromagnetic Theory classroom
}

\author{
Andrew J. Berger*a \\ ${ }^{a}$ The Institute of Optics, University of Rochester, Rochester, NY 14627
}

\begin{abstract}
Electromagnetic Theory is a required junior-year course for Optics majors at the University of Rochester. This foundational course gives students their first rigorous exposure to electromagnetic vector fields, dipole radiation patterns, Fresnel reflection/transmission coefficients, waveguided modes, Jones vectors, waveplates, birefringence, and the Lorentz model of refractive index.

To increase the percentage of class time devoted to student-centered conceptual reasoning and instructor feedback, this course was recently "flipped". Nearly all of the mathematically-intensive derivations were converted to narrated screencasts ("Khan Academy" style) and made available to students through the course's learning management system. On average, the students were assigned two 10-15 minute videos to watch in advance of each lecture. An electronic survey after each tutorial encouraged reflection and counted towards the student's participation grade. Over the past three years, students have consistently rated the videos as being highly valuable.

This presentation will discuss the technical aspects of creating tutorial videos and the educational tradeoffs of flipping a mathematically-intensive upper-level course. The most important advantage is the instructor's increased ability to identify and respond to student confusion, via activities that would consume too much time in a lecture-centered course. Several examples of such activities will be given. Two pitfalls to avoid are the temptation for the instructor not to update the videos from year to year and the tendency of students not to take lecture notes while watching the videos.
\end{abstract}

Keywords: electromagnetic theory, optics, flipped classroom

\section{INTRODUCTION}

Undergraduates at the University of Rochester who major in Optics or Optical Engineering are required to take a class entitled "Electromagnetic Theory", typically in their junior year. Prior to this class, the average student has taken prerequisite classes in Geometrical Optics and Interference \& Diffraction, along with multi-semester introductory sequences in Math and Physics. The students therefore arrive with significant preparation in both optics concepts and mathematical formalism.

In the Electromagnetic Theory course, many properties of optical fields and materials that were postulated in previous courses are now derived from more fundamental starting points. Examples include the radiating electric field of an oscillating electric dipole (from which plane waves are then obtained at large distances), the wavelength dependence of refractive index (from the Lorentz model of a damped, driven atomic oscillator), and the Fresnel reflection and transmission laws at planar interfaces. All of these derivations are classic ones that blend conceptual and mathematical reasoning.

This paper describes an attempt to make students engage with these classic derivations prior to class, freeing up time in the classroom to administer assessment activities and offer just-in-time mini-lectures. To set the stage for this attempt, the five statements below are offered as postulates about the students in this particular course:

1. Students must be exposed to derivations - it's only a question of where to put them.

2. Students have no time to reflect upon a derivation while it's being delivered.

3. Students do not interrupt live derivations.

4. Students who will not read book chapters will watch video tutorials.

5. Live derivations are not as clean as recorded ones.

The instructor chose the standard "flipped classroom" approach of providing videos that the students were supposed to watch before coming to class. The following sections of this paper describe the technical details of creating these videos, the way they were integrated into the class, and some discussion of the benefits and shortcomings.

14th Conference on Education and Training in Optics and Photonics: ETOP 2017, edited by Xu Liu,

Xi-Cheng Zhang, Proc. of SPIE Vol. 10452, 104522N · C 2017 ICO, IEEE, OSA, SPIE

CCC code: $0277-786 \mathrm{X} / 17 / \$ 18 \cdot$ doi: $10.1117 / 12.2269783$

Proc. of SPIE Vol. $10452104522 \mathrm{~N}-1$ 


\section{TECHNICAL CREATION OF VIDEOS}

Over the course of two academic Fall terms (2014 and 2015), approximately forty videos were recorded. They were in the style of "Khan Academy" videos (see www.khanacademy.org): a video capture of a computer screen region, usually a simple canvas on which handwritten pen lines are drawn in real time, along with audio narration, with a length rarely exceeding 15 minutes. In some of the videos, computer animations were also included. The videos are publicly available on YouTube and readily found by searching "Electromagnetic Theory" and "Berger". As an example, the link to a particular video (on refractive index) is

https://www.youtube.com/watch?v=NAALII7THHM.

Creating videos requires making some technical choices. The particular ones used for this project are shown below.

\subsection{Stylus}

A Wacom metal tablet and stylus were used to control the mouse for drawing on the computer screen. The tablet cost approximately $\$ 180$. It connected by USB to the computer and acted as a duplicate touchpad. Although it takes at least one extended test session to get accustomed to writing naturally on the metal surface, it eventually becomes automatic and is much more nuanced than finger strokes on a typical laptop touchpad.

Touchscreen displays with styluses (e.g. the Microsoft Surface) continue to improve and are viable alternatives to the metal tablet. Compared to such touchscreens, the metal tablet does have some advantages. For example, the Wacom metal tablet can operate in a mode where it is completely insensitive to contact with the user's hand. Using a stylus directly on a touchscreen typically produces one or more inadvertent strokes per recording session due to the user's hand (when the pen tip is too far away from the screen to override it). Another minor advantage is that, when writing on a metal tablet, one's hand does not obscure any of the existing writing on the actual screen.

\subsection{Drawing program}

The canvas for the drawings in these videos was the work area of the free drawing program SmoothDraw (www.smoothdraw.com). This simple program provides several different colors and pen thicknesses. Importantly, it enables several color choices to be mapped to function keys. The user's non-writing hand can therefore change the pen color without the pen having to leave the canvas, which cuts down on wasted time.

\subsection{Recording program}

Screencasts were recorded using the free version of the program ActivePresenter

(https://atomisystems.com/activepresenter/free-edition). There are many programs available that can record screens with audio input. ActivePresenter has a few key properties that were not found combined in any other free program. First, it enabled recording from a subregion of the screen. This meant that the unimportant control areas surrounding the SmoothDraw canvas did not have to appear in the recording; one could record solely from the actual drawing area, for a cleaner effect. The subregion also meant that it was easy to place other needed windows (e.g. for computer simulations) outside the recording area and drag them in when needed, rather than having to "unhide" them from the background.

A second important feature of ActivePresenter is that it provides a rich editing environment for the recording. Most simply and almost most crucially, it makes it easy to delete sections of the video. There were three separate ways in which the deletion process improved the final video over the real-time original:

1. Pauses. Sometimes during a recording, the instructor needed a few seconds to gather his thoughts. Rather than having to decide whether to hit the pause button, the instructor could simply focus on getting back on track, knowing that the pause could be deleted later.

2. Mistakes. Sometimes the instructor realized in the middle of a recording that he wanted to back up and fix the most recent thing he had said or written. Rather than having to do a second take and splice it in, the instructor could simply pause, erase part of the screen, and start over, knowing that the "goofup" section could be deleted later. 
3. Long mathematical expressions. Sometimes in a derivation there is a line of math that takes several seconds to write but isn't worth narrating while doing so (especially if much of it is the same as the previous line). In such cases, the instructor learned to say, "I'll just make this next line appear," and then wrote the line of math without talking, knowing that the writing time could be deleted in the final version.

The free version of ActivePresenter did place a watermark logo in the upper right-hand corner of the videos. The area blocked was not a big problem.

\subsection{Dissemination}

Videos were exported from ActivePresenter to .MP4 format and uploaded to YouTube. On both computers and smartphones, YouTube viewers currently enable playback at higher speeds such as $1.5 \mathrm{X}$ and $2 \mathrm{X}$. This creates a win-win situation: if the instructor speaks slowly and carefully to avoid mistakes, the sped-up audio isn't too fast for the students' comprehension, so they can watch (and re-watch) the video in less time.

Links to the videos were embedded in online quizzes that were disseminated to the students via the course's Learning Management Software (LMS) system. Each quiz revealed the link and required that the students answer one to three short questions related to the material in the video. The students knew that taking the quizzes affected their participation grade.

\section{RESULTS AND DISCUSSION}

\subsection{Use of classroom time}

With the mathematical derivations shifted to online preparation, class would now start with a challenge question inspired by one of the assigned videos. Students would work at their desks for a few minutes, vote on the correct answer (if appropriate), and hold a class discussion of the correct answer. This enabled the instructor to assess how well the class was utilizing the relevant material from the video. Just-in-time mini-lecturing would follow as needed, often with a screenshot of the video shown on the projector to remind students of the video content. When the screenshot was shown, students would frequently come up with new questions about the video.

The remainder of most class periods was spent working on parts of the current problem set, with chances for just-in-time teaching as needed. This was the explicit contract explained on the first day: that the flipped classroom would not increase their time commitment to the course, because some of the lecture time "saved" by watching a video would be "given back" in class to make progress on the homework.

\subsection{Student compliance and feedback}

Typically, more than $90 \%$ of students completed the online quiz for any given video. That does not prove that the students watched the video itself; they could have answered the questions without watching, since participation scores were not graded for correctness. No direct metrics were gathered from YouTube on the number of unique users who watched the videos.

Student feedback on the videos was strongly positive. At mid-semester, students completed a three-question survey: "What should this class START doing? STOP doing? KEEP doing?" The majority of the responses to "KEEP doing" focused on the videos. Students said that the videos were helpful and that they valued the way the video content was linked to in-class problems and discussions. They also mentioned that they liked having the videos around so they could review them prior to the two midterms and the final exam.

\subsection{Pitfalls}

Converting derivations to online tutorials creates some problems. Obviously there is the nontrivial time commitment associated with creating the videos; in this instructor's hands, it took at least 2 hours just to record and edit a 15-minute final video, not including the preparation before the recording. This work is of course offset by the fact that the video can be reused in subsequent years. There are, however, other challenges that are more systemic.

1. Because the online videos are preserved and accessible at any juncture during the rest of the semester, students are less likely to take notes on videos than on in-class lectures. The students need to be explicitly told to treat the tutorial videos as they would live lectures, in terms of note-taking to promote sense-making. 
2. The instructor has to be judicious about revisiting too much of the video material during class time, even if it seems warranted by widespread student difficulty with challenge questions. Student feedback indicates that this is seen as "double lecturing" and a violation of the flipped classroom contract.

3. Once a video has been recorded and edited, it is tempting to leave it intact in future years rather than make minor changes in response to a new year's class. The instructor must, at a minimum, review the videos personally each year to catch potential points of confusion, such as a change in how variables are named.

4. Putting content online makes it easier for some students to justify skipping class; for a certain type of strong student, it makes it easier to get a $\mathrm{B}+$ or higher without coming to class as often. This can bruise the ego of an instructor. However, if a student is able to do well on all credit-bearing aspects of a class, the instructor cannot (or should not) complain about the path the student is taking.

\section{CONCLUSION}

The five postulates in the Introduction section all played a role in flipping this particular Electromagnetic Theory class. There was no thought of abandoning the derivations (Postulate 1), so the only way to get more time to work on problems in class was to push the derivations outside of class. Videos were chosen as the vehicle because the instructor believed that static documents (book chapters, course notes) would not be perused as closely (Postulate 4). Although the online delivery meant that students could not ask questions during the videos, this was no different in practice from the way students behaved during previous years' live derivations (Postulates 3). By getting the students to reflect upon the material ahead of time, there was in fact a greater chance of eliciting useful questions from them in the classroom, where the instructor has the unique ability to provide targeted answers (Postulate 2). And lastly, producing a succinct and mistake-free video tutorial via the editing process is, at least for this instructor, significantly less time-consuming than providing the same quality of performance in live mode in a single year (Postulate 5), let alone in multiple years. 